\section{ICU: Neuromuskulärer Index zur Erfassung der Atemarbeit noch zu ungenau}

Jansen D et al. Estimation of the diaphragm neuromuscular efficiency index in mechanically ventilated critically ill patients. Critical Care 2018; doi:10.1186/s13054-018-2172-0

Dysfunktionen des Zwerchfells sind häufige Komplikationen einer Langzeitbeatmung und können die Rehabilitation erheblich beeinträchtigen. Um Dystrophie oder Erschöpfung des wichtigsten Atemmuskels rechtszeitig erkennen zu können, kommt heute die Messung des sogenannten neuromuskulären Effizienzindexes (NME) zu Einsatz. Jansen und ihr Team haben nun getestet, wie zuverlässig einzelne Messungen im Rahmen eines Okklusionsmanövers sind.

Die Langzeitbeatmung stellt auch für erfahrene Intensivmediziner immer wieder eine Herausforderung dar. So können eine überassistierte Beatmung zur Zwerchfellatrophie und eine zu geringe Unterstützung zu Muskelerschöpfung führen. Am Ende entsteht dann oftmals eine ernsthafte Dysfunktion des wichtigsten Atemmuskels, die wiederum die Rehabilitation der Betroffenen deutlich beeinträchtigen kann und im schlimmsten Fall zu Langzeitschäden führt. Um nun die Arbeitsleistung des Zwerchfells während der Beatmung erfassen zu können, hat sich in letzter Zeit der sogenannte neuromuskuläre Effizienzindex (NME) durchgesetzt. Durch wiederholte Messungen im Rahmen von endexspiratorischen Okklusionsmanövern soll dabei der Gesamtdruck durch alle beteiligten Atemmuskeln gemessen werden.

Da zu diesem Verfahren bislang größere Studien fehlen, haben Jansen und ihre Kollegen nun im Rahmen einer prospektiven Kohortenstudie die Messgenauigkeit der Erfassung des NMEs genauer untersucht. Die Studie fand auf einer Intensivstation eines Uniklinikums statt. Geeignete Patienten erfüllten folgende Kriterien:
- Alter ab 18 Jahren,

- mechanische Beatmung,

- Katheter zur Messung der elektrischen Zwerchfellaktivität.

Die Messung des NMEs erfolgte jeweils im Rahmen eines endexspiratorische Okklusionsmanövers. Bei jedem Patienten führten die Forscher in einem Zeitfenster von 72 Stunden zu 4 unterschiedlichen Messzeitpunkten im Abstand von jeweils 12 Stunden innerhalb einer Minute 5 einzelne NME-Messungen durch. Sie wollten vor allem die intraindividuelle Variabilität bestimmen und ermittelten dazu einen entsprechenden Variabilitätskoeffizienten.

\section{Hohe intraindividuelle Variabilität}

31 Intensivpatienten unter mechanischer Beatmung erfüllten die Kriterien, die Daten aus insgesamt 459 Okklusionsmanövern gingen in die finalen Analysen mit ein. Bei 19 Patienten konnten nicht zu allen vorab festgelegten Messzeitpunkten alle Messungen durchgeführt werden. Häufige Gründe dafür waren Extubation, Agitation oder vorzeitiges Versterben.

Die Studienteilnehmer waren im Durchschnitt 69 Jahre alt, 22 von ihnen männlich. Als häufigste Gründe für eine invasive Beatmung nannten die Autoren Pneumonie, postoperativen Status oder Trauma mit jeweils $42 \%, 26 \%$ und $23 \%$. Die mittlere Beatmungsdauer konnte auf 24 Tage beziffert werden.

Die Messergebnisse blieben im zeitlichen Verlauf konstant, die Forscher mussten allerdings eine hohe intraindividuelle Variabilität mit einem Variabilitätskoeffizienten von durchschnittlich 63,4\% feststellen. Wurden zu einem Messzeitpunkt die drei Ergebnisse mit der geringsten Variabilität zusammengenommen, verbesserte sich der Variabilitätskoeffizient auf $29,8 \%$.

Der NME zum Messzeitpunkt T0 zu Beginn des 72-stündigen Beobachtungszeitraumes lag bei $1,22 \mathrm{cmH}_{2} \mathrm{O} / \mu \mathrm{V}$ mit einer Standardabweichung von
$0,86 \mathrm{~cm}_{2} \mathrm{O} / \mu \mathrm{V}$ und einem Variabilitätskoeffizienten von 82,6\%. Ein Patient mit einem $\mathrm{NME}$ von $1,22 \mathrm{cmH}_{2} \mathrm{O} / \mu \mathrm{V}$ hat also mit einer Wahrscheinlichkeit von 95\% einen NME zwischen 2,22 und $0,22 \mathrm{cmH}_{2} \mathrm{O} / \mu \mathrm{V}$. In ihrem Diskussionsteil und Fazit beurteilen Jansen und ihre Kollegen daher insbesondere die einfache Messung des NME als sehr ungenau.

\section{FAZIT}

In dieser prospektiven Kohortenstudie zeigten wiederholte Messungen des neuromuskulären Effizienzindexes (NME) zur Beurteilung der Atemarbeit von beatmeten Intensivpatienten eine sehr hohe intraindividuelle Variabilität. Obwohl sich der NME nach Meinung der Autorinnen/Autoren prinzipiell zur Früherkennung einer Dysfunktion des Zwerchfells eignen würde, halten sie eine Weiterentwicklung der Auswertungssoftware mithilfe großer Studien für nötig.

Dipl.-Psych. Annika Simon, Hannover 